\title{
Powdered Activated Charcoal Tracing in Hand Hygiene Training and Compliance Assessment During the COVID-19 Pandemic
}

This article was published in the following Dove Press journal: Risk Management and Healthcare Policy

\section{Guo Feng \\ Han Jun \\ Gitonga Elaine \\ Shen Haitao (D)}

Department of Emergency Medicine, Shengjing Hospital of China Medical University, Shenyang, I I0004, People's Republic of China
Correspondence: Shen Haitao

Tel +86-24-966I5-64II3

Email pianzhang6@163.com
Objective: Because of the COVID-19 pandemic there has been a significant increase in the prevalence of nosocomial infections. As a result, we sought to find an effective, efficient and safe way to train healthcare workers on proper hand washing techniques. We used powdered activated carbon (PAC) as a tracer to visually display hand washing defects after the hand washing process. The real-time visual assessment of the efficacy of the hand washing technique aided in the immediate correction of errors, and this definitively improved hand hygiene techniques of the interns.

Methods: Clinical interns at the emergency department of Shengjing Hospital were included in this study and received training in relation to the six-step hand-washing technique developed by the World Health Organization (WHO). The subjects' hand-washing defects or faults were traced using PAC and corrected accordingly. Acceptance of the PAC tracing method by the interns, and its safety, were both assessed using a questionnaire survey.

Results: The results indicated that the back of the hands, fingers, and the wrists were prone to hand-washing defects. The hand-washing defects were significantly reduced after targeted corrections by the trainers. Subjects reported satisfactory acceptance toward the PAC tracing method and the method was relatively safe for subjects.

Conclusion: The PAC tracing method can visually display hand-washing defects and significantly improve the effectiveness of hand-washing training.

Keywords: hand hygiene, COVID-19, infection control

\section{Introduction}

COVID-19 (coronavirus disease 2019) is a highly transmissible disease caused by a pathogenic coronavirus that emerged in late 2019 and is still prevalent to date. Virus transmission occurs via direct (deposited on persons) or indirect (deposited on objects) contact and airborne (droplets and aerosols) routes. Because of its high transmission efficiency, COVID-19 extensively spread throughout the world, becoming a global pandemic. This ongoing global pandemic has caused significant burden on healthcare systems around the world. ${ }^{1}$ During the initial phase of the pandemic, medical personnel in the front lines experienced extreme physical (fatigue due to increased workload and working hours) and psychological stress (stress, anxiety and depression). As the pandemic grew rampant, many healthcare centers experienced rapid dwindling of personal protective equipment (PPE) and gloves. The shortages contributed to an increased fear and risk of COVID-19 transmission, especially to healthcare workers. These factors increased the associated burden in relation to the 
possibility and prevalence of nosocomial COVID-19 infections. ${ }^{2}$ This imposed burden on all healthcare workers definitively influences the performance of medics in relation to patient management; an increase in both physical and psychological stressors not only reduce the efficiency of treatment for patients but also leads to a substantial increase in mortality. ${ }^{3}$

Nosocomial infections or healthcare associated infections are infections that occur in patients and healthcare workers during medical care and are a major cause of increased morbidity and mortality in medicine. Nosocomial pathogens include bacteria, viruses, and fungal parasites. There are many causes of HAI's; contaminated hospital equipment, air droplets, but the major cause is person-to-person transmission, which is mainly the spread of pathogens via the hands of health care personnel to the patients or even to the health care personnel themselves. ${ }^{4}$ The different pathogens carried on the hands are highly consistent with nosocomial infection pathogens that may cause healthcare associated infections. ${ }^{5}$ Infection by physical contact is also one of the main pathways for transmission of COVID-19. Hand hygiene and decontamination can be achieved through two fundamental techniques: (1) the waterless technique (use of alcohol based hand rub) and (2) normal hand washing. Both hand hygiene techniques decimate pathogens on the skin, therefore, reducing the likelihood of pathogen transfer, ie, coronaviruses and have therefore proven to be effective infection-control measures. ${ }^{6,7}$

Hand-washing is an important part of hand hygiene and refers to the process of washing hands with soap (medicated soap or normal plain soap) and running water to remove dirt, debris, and pathogens from the hands. ${ }^{8,9}$ In order to ensure the beneficial effects of hand-washing, the WHO has formulated guidelines for hand-washing training. Different research institutions have endeavored to evaluate hand-washing technique in order to determine the proper implementation and technique associated with hand hygiene. However, the pattern recognition methods used in the evaluations mainly focused on the execution and technique of handwashing, and they did not accurately determine whether the hands were effectively cleaned. ${ }^{10-12}$ Other methods used in the evaluation of the degree and efficacy of hand hygiene are through the use of phosphors and ultraviolet rays; these methods increase the difficulty and risk of training, making these methods unsuitable for extensive application. ${ }^{13} \mathrm{~A}$ traditional hand colony measurement method can also evaluate the effects of hand-washing; however, it is beset by lagging evaluation of effect and poor visibility, which makes it relatively unsuitable for hand-washing training. ${ }^{14,15}$ Considering the above, we attempted to use direct visualization to trace the efficiency of the hand-washing process using PAC. In order to better understand the process, we made a schematic presentation, as shown in Figure 1. Powdered activated charcoal is readily available in hospitals or other health care facilities and can be used as an assessment tool in the evaluation of effectiveness and efficacy of hand-washing and as a result improve training outcomes. ${ }^{16}$

\section{Materials and Methods}

\section{Research Subjects and Ethics Approval}

A total of 189 clinical interns at the Emergency department of Shengjing Hospital were enrolled in the study between July 2020 and October 2020 after delayed resumption to school due to the ongoing COVID-19 pandemic. Subject characteristics including age, gender and

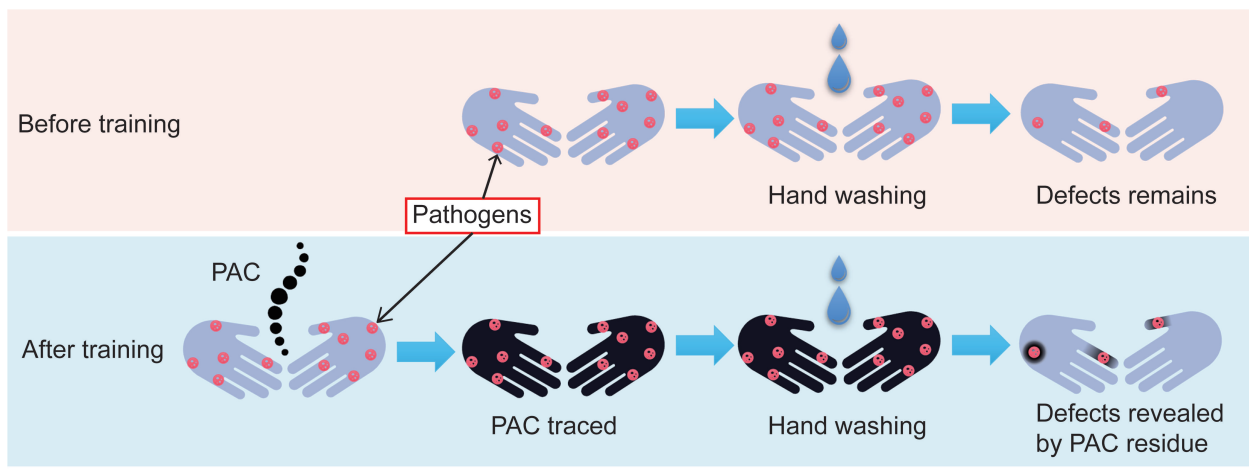

Figure I The defects were revealed by PAC residue after the training. 
Table I Subject Characteristics

\begin{tabular}{|c|c|c|c|c|c|c|c|}
\hline \multirow[t]{2}{*}{ Items } & \multicolumn{2}{|c|}{ General Information } & \multicolumn{5}{|l|}{ Group } \\
\hline & $\begin{array}{l}\text { Age ( } \chi \\
\pm \text { sd) }\end{array}$ & $\begin{array}{l}\text { Gender } \\
\text { (Male) }\end{array}$ & $\begin{array}{l}\text { Clinical } \\
\text { Medicine }\end{array}$ & $\begin{array}{l}\text { Medical } \\
\text { Imaging }\end{array}$ & Pediatrics & $\begin{array}{l}\text { International } \\
\text { Students }\end{array}$ & Total \\
\hline Ratio n (\%) & $21 \pm 1.45$ & $78(4 \mid .3)$ & I0I (53.4) & $29(15.3)$ & $31(16.4)$ & $28(14.8)$ & $\begin{array}{l}189 \\
(100)\end{array}$ \\
\hline
\end{tabular}

medical specialties in terms of their current departmental rotation are shown in Table 1.

The Inclusion criteria: Medical student interns rotating in the hospital. Exclusion criteria: production interns, resident and accompanying doctors, doctors engaged in advanced studies, and those with damaged skin (dermal injuries, skin abrasions or an ongoing infection on the hands). All subjects volunteered to participate for the study. Results from the questionnaire survey were blinded to study investigators. This study was approved by the Ethics Committee of Shengjing Hospital of China Medical University (2020PS027J), and the principles of Helsinki Declaration were followed. All participants were informed as to the purpose of this study, and we confirm that consents were received from the participants.

\section{Materials}

Analytical pure PAC produced by Chengdu Kelong Chemicals.

\section{Tracing and Hand-Washing Method}

Prior to the hand washing, all subjects received education and training on the importance of hand hygiene as a tool against nosocomial infections and also the WHO six-step hand-washing method. Ensure that participants' hands are completely clean (Figure 2A). All subjects were provided with $3 \mathrm{~g}$ powdered activated charcoal. They were then advised on the manner at which to apply and spread the PAC, which was going to be used as a tracer. All the subjects had to ensure that the PAC was evenly distributed on the skin and was coating all the surfaces of to their hands and wrists (Figure 2B). The interns proceeded to washing their hands following the WHO six-step hand-washing method (Figure 3). The entire process took 40-60 seconds. The subjects were asked to wash their hands with running water. The hands were not wiped dry so as to not wipe off the remaining PAC residues. The effectiveness of the handwashing process was visually evaluated by the trainers, and the presence or absence of PAC residues on both hands was recorded.

\section{Evaluation of Hand-Washing Effectiveness}

According to the six steps in the WHO hand-washing method, the hand is divided into six parts, including the palm, back of the hand (including the volar side), fingers, back of the fingers, finger-tips and nails, and thumb. In addition to these six parts, the wrist was also considered for the visual evaluation. A visual assessment of these seven parts was undertaken after the hand washing process. The parts of the hand that had clear PAC residues were recorded (Figure 2C).

For the parts of the hand that PAC residues could not be accurately identified after hand-washing, white cotton swabs were used to scrape the surface within the areas for further observation (Figure 2C). The parts of the hand with PAC residues were defined as having hand washing defects or faults.

\section{Improvement and Assessment of Hand-Washing}

According to the parts of the hands that had PAC residues, the hand-washing defects of each subject were determined and recorded as pre-training defects. Re-training was targeted at the steps associated with defects to help participants clean their hands completely (Figure 2D). Assessment of hand-washing effectiveness was conducted again using the PAC tracing method. The parts of the hands that still had PAC residues were recorded as posttraining Defects I. A day after the re-training, the handwashing technique was re-assessed for the third time and parts of the hands that had PAC residues were noted and recorded as post-training Defects II.

\section{Acceptance Assessment}

The training method was assessed using a questionnaire to evaluate the subjects understanding of the WHO six step hand-washing protocol. The questionnaire provided feedback on the errors in technique for the different subjects 


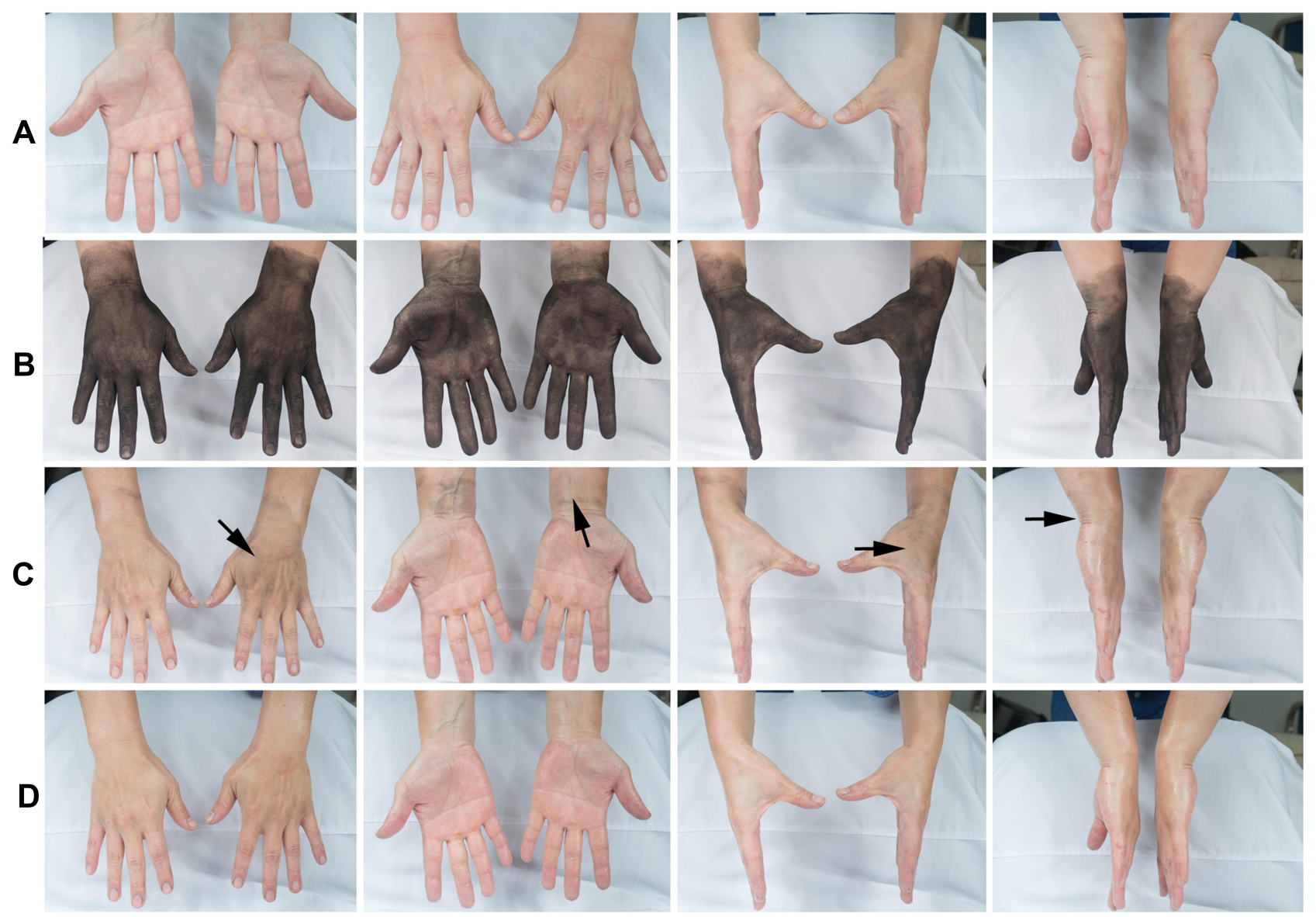

Figure 2 PAC tracing method can visually display hand-washing defects. (A) Before application; (B) After application; (C) Hand-washing defects revealed by PAC residue (Arrow); (D) No PAC residues after careful washing.
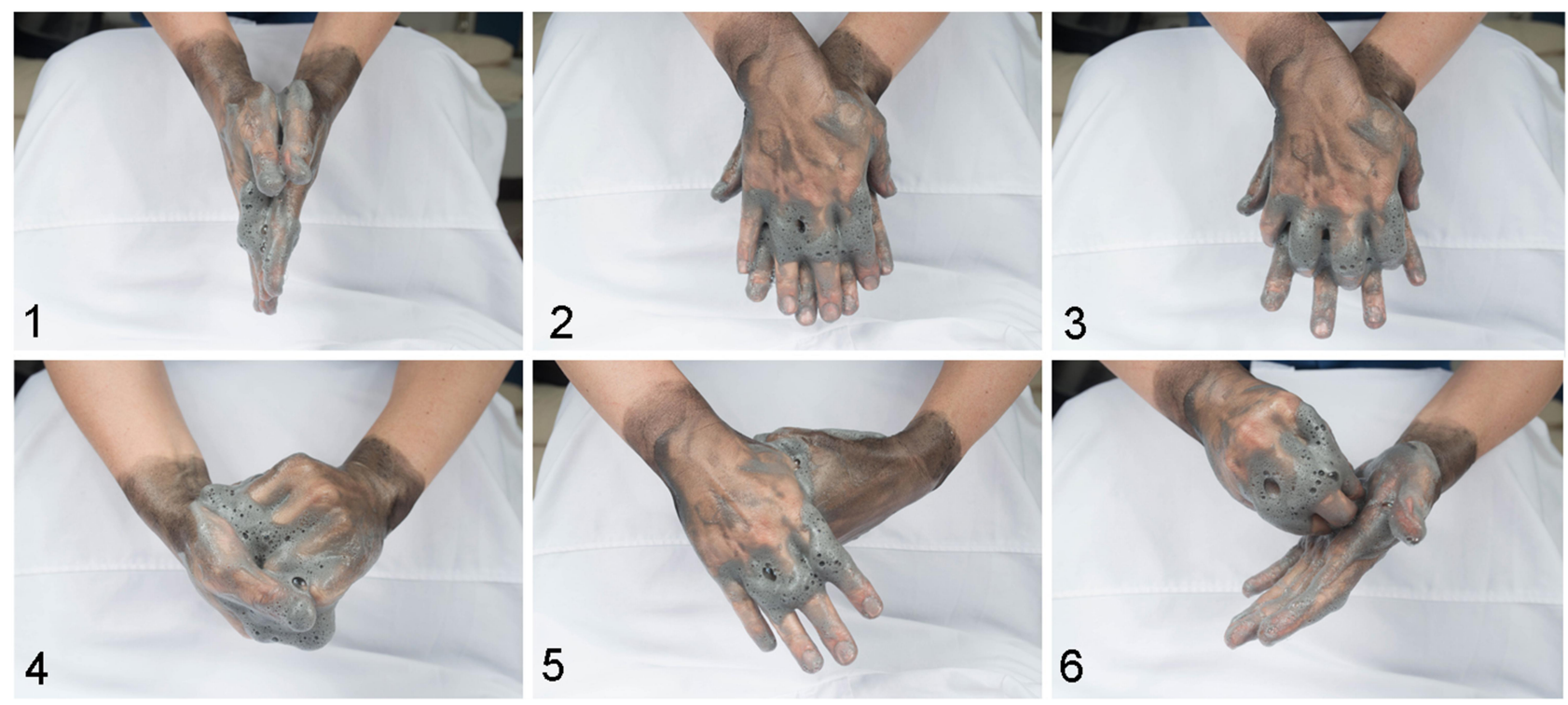

Figure 3 Six-step hand-washing after applying PAC. 
Table 2 The Hand Washing Defects Reduced After Training

\begin{tabular}{|l|l|l|l|l|l|}
\hline Part & Before Training & First Washing After Training & Second Washing After Training & Chi-Square Value & P \\
\hline Hand back & $107(56.6)^{\mathrm{ab}}$ & $34(18.0)^{\mathrm{a}}$ & $48(25.4)^{\mathrm{b}}$ & 73.22 & $<0.00 \mathrm{I}$ \\
Finger back & $82(43.4)^{\mathrm{ac}}$ & $15(7.9)^{\mathrm{ab}}$ & $37(19.6)^{\mathrm{bc}}$ & 75.257 & $<0.00 \mathrm{I}$ \\
Finger side & $41(21.7)$ & $27(14.3)$ & $30(15.9)$ & 4.725 & 0.094 \\
Finger tip & $25(13.2)$ & $18(9.5)$ & $30(15.9)$ & 3.159 & 0.206 \\
Palm & $34(18.0)$ & $28(14.8)$ & $35(18.5)$ & 1.483 & 0.476 \\
Thumb & $16(9.5)$ & $12(6.3)$ & $23(12.2)$ & 4.133 & 0.127 \\
Wrist & $78(41.3)^{\mathrm{ac}}$ & $21(11.1)^{\mathrm{ab}}$ & $50(26.5)^{\mathrm{bc}}$ & 47.784 & $<0.00 \mathrm{I}$ \\
\hline
\end{tabular}

Note: ${ }^{a, b, c}$ Differences between groups are statistically significant.

that were displayed by areas with PAC residues; this was used in the re-training process so as to help improve the effectiveness of hand-washing. The feedback provided also assisted in grading the convenience of the learning process, and highlight any presence of adverse reactions. Survey items were assessed according to three grades (superior, middling, and inferior). The subjects were given a quick response code (QR code) for reporting the presence or absence of adverse reactions like redness, irritation, pain, and blisters on the hands. The collection period for adverse reactions was 1 month.

\section{Statistical Analysis}

Nominal variables are expressed as $\mathrm{n}(\%)$, and continuous variables as $\mathrm{X} \pm \mathrm{d}$. Cochran's $\mathrm{Q}$ test was used to compare the results before and after hand-washing, and Dunn's test (subject to Bonferroni correction) was used for pairwise comparison. Adjust. $P$ or $P<0.05$ indicated that a difference was statistically significant. The survey results are expressed by a frequency distribution diagram.

\section{Results}

\section{Hand-Washing Defects}

Of the 189 subjects, there were no statistically significant differences in hand-washing defects between the palm, the spaces in-between the fingers, finger-tip, and thumb ( $\mathrm{P}=0.476, \mathrm{P}=0.094, \mathrm{P}=0.206, \mathrm{P}=0.127$ ), however significant hand-washing defects were observed on the back of the hand, back of the fingers and wrists $(\mathrm{P}<0.001)$. Handwashing defects on the back of the hand were significantly different between pre-training defects, post-training Defects I, and post-training Defects II, however no statistical differences were observed between post-training Defects I and post-training Defects II. Hand-washing defects on back of the fingers and wrists were significantly different between pre-training defects and post-training Defects I (19.6\% vs $7.9 \%$ ), and between pre-training defects and post-training Defects II (26.5\% vs $11.1 \%)$ respectively. Post-training Defects I had a larger incidence of hand-washing defects than the post-training Defects II. See Table 2 for details and Figure 4 for a specific distribution.
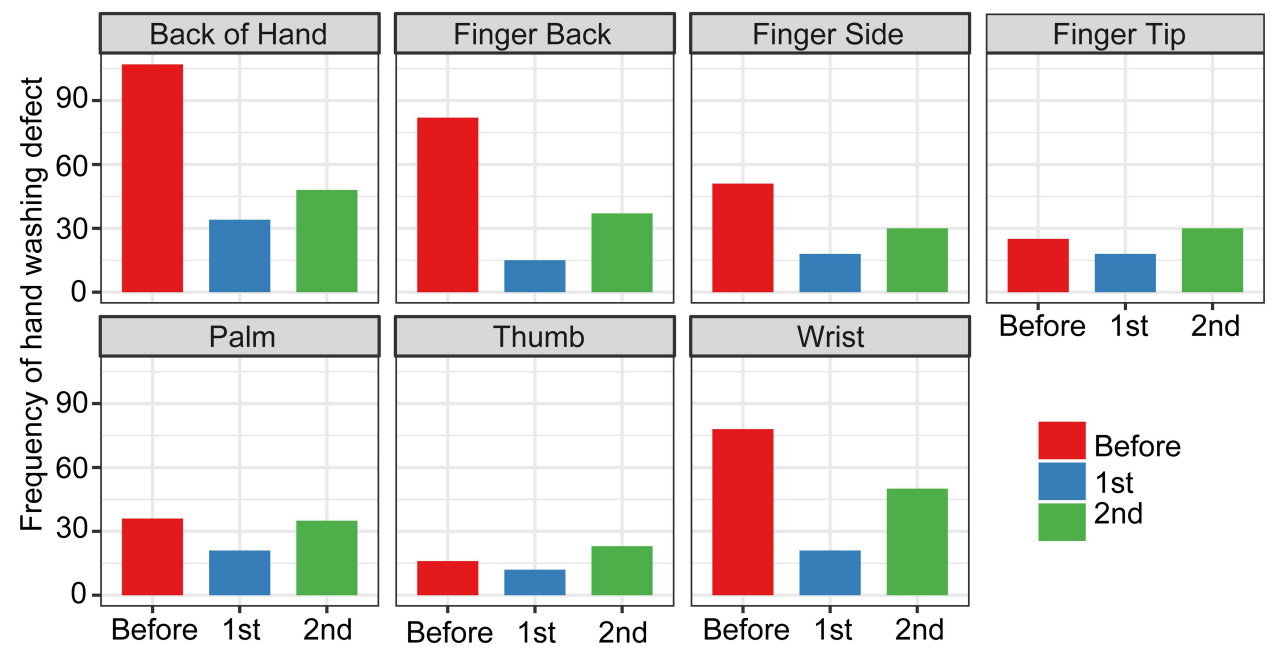

Figure 4 PAC tracing method improved hand washing. 


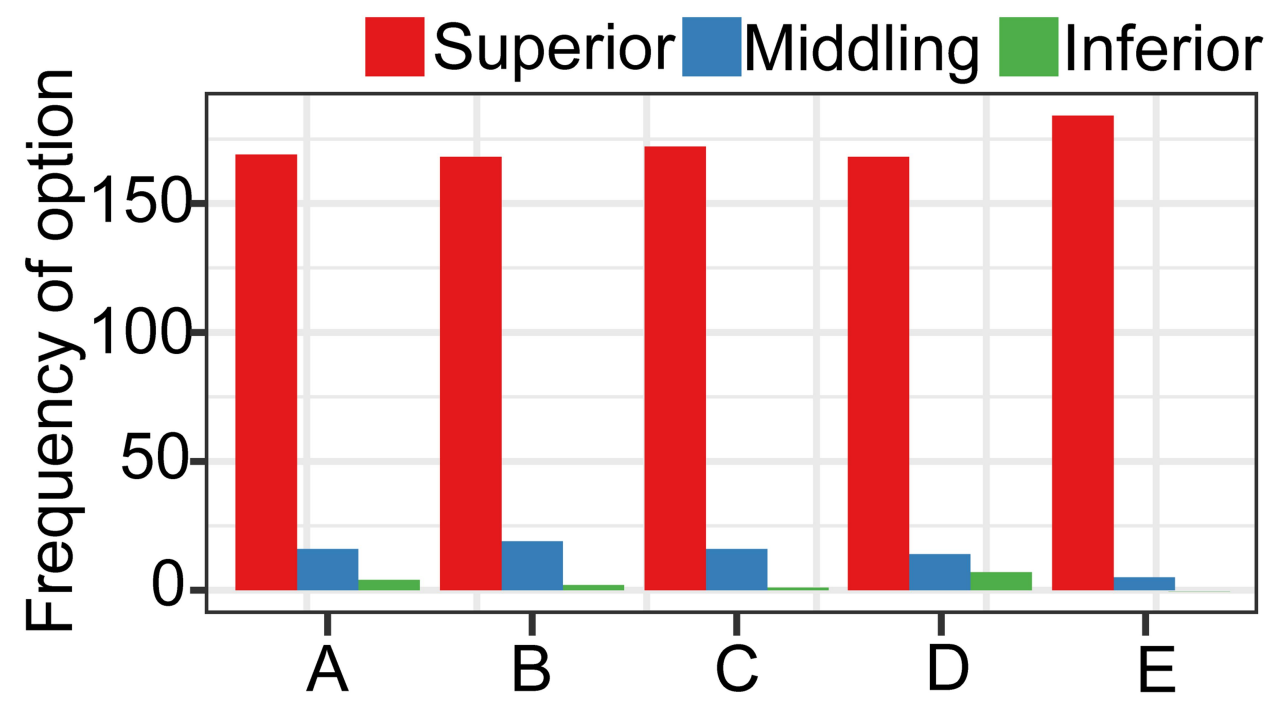

Figure 5 The PAC tracing method is well received. (A) An understanding of the hand-washing progress; (B) Direct feedback on the effect of hand-washing; (C) Help to improve the effect of hand-washing; (D) Convenience of learning process; (E) Adverse reaction.

\section{Acceptance Survey Results}

Results showed that most of the subjects considered the training method "superior", and had no reported adverse reactions (Figure 5).

\section{Discussion}

The World Health Organization describes "hygiene" as the conditions and practices that help maintain health and prevent the spread of diseases. Healthcare-associated infections are a major cause of morbidity and mortality in medicine, and hand hygiene is the main undisputed weapon against nosocomial pathogens and hospitalacquired infections (HAI) or nosocomial infections. Despite extensive research on nosocomial infections and creation of hand hygiene techniques to prevent and combat hospital-acquired infections (HAI), they still remain a major global medical concern, especially now with the current rampant COVID-19 pandemic.

The coronavirus disease (COVID-19) has multiple routes of transmission and an extremely strong ability to spread. It has been reported to be able to survive for over 72 hours on the surfaces of various objects, ${ }^{17,18}$ thus increasing the risk of nosocomial COVID-19 infections. There has been a surge in nosocomial infections in many countries, ${ }^{2,19,20}$ and this is partially attributed to the increase in transmission of nosocomial COVID-19 infections; however, it has also been suggested that insufficient preventative measures adopted by medical staff also contributed to this increase. Studies have indicated that contact transmission is one of the primary methods of spreading this disease, ${ }^{3}$ and hand hygiene is considered an effective way to prevent contact transmission. ${ }^{21,22}$ Lake MA summarized a variety of methods to stop SARS-CoV -2 transmission, including hand washing, and pointed out that in addition to generally avoiding hand-face contact, hand washing is the first choice for hand disinfection, especially after returning home from public places and having close contact with any potential infected persons. ${ }^{3}$ Researchers also provided data on the role of hand hygiene in blocking SARS-CoV-2 transmission. Two Original formulations were recommended by WHO. Formulation I consists of $80 \%$ (vol $/ \mathrm{vol}$ ) ethanol, $1.45 \%$ ( $\mathrm{vol} / \mathrm{vol}$ ) glycerol, and $0.125 \%$ (vol $/ \mathrm{vol})$ hydrogen peroxide. Formulation II consists of $75 \%$ (vol/vol) 2-propanol, $1.45 \%$ (vol $/ \mathrm{vol}$ ) glycerol, and $0.125 \%$ (vol/ $/ \mathrm{vol}$ ) hydrogen peroxide. ${ }^{9}$ Hand disinfection through the use of alcohol based hand rub (ABHR) containing ethanol and isopropyl alcohol can inactivate SARS-COV-2 within 30 seconds at a concentration of $30 \%-80 \%{ }^{5}$ It has been previously reported that hand hygiene training is not standardized or has poor compliance, ${ }^{22,23}$ and nosocomial infections caused by hand hygiene defects have been a longstanding problem in the medical system. ${ }^{24,25}$ Therefore, in order to prevent nosocomial infections and COVID-19, correct execution of hand hygiene methods, be it through hand washing or the use of $\mathrm{ABHR}$, is an effective measure in infection control processes. ${ }^{20,25}$

Studies have shown that hand hygiene can be improved through various interventions, ${ }^{26,27}$ of which hand-washing training is of the utmost importance. ${ }^{28}$ 
Evidence suggests that good training can significantly improve hand hygiene of medical practitioners, and their compliance in implementing of proper hand hygiene practices. ${ }^{29}$ However, most of the existing hand-washing training methods only evaluate hand-washing effectiveness through delayed bacterial hand colony count results, failing to correct hand-washing defects in actual time. $^{14,15,30}$ Therefore, visually displaying hand-washing defects as part of training in real-time may greatly improve the quality of training. ${ }^{31}$ For this reason, we examined the PAC tracing method as a tool to provide an immediate assessment of hand-washing defects in an effort to improve the quality of hand-washing training. In order to confirm that the subjects remembered the proper technique, after the initial training, we added an additional assessment time point (the second day after the training). The current results suggested that the hand-washing defects, on the first day and the second day after the training, were significantly reduced as compared to hand washing defects before the training.

Before training, three parts of the hand (back of the hand, back of the fingers and the wrists) had a high incidence of hand-washing defects, reaching 56.6\%, 43.4\%, and $41.3 \%$, respectively. Since the WHO six-step handwashing method does not involve wrist washing, a sevenstep hand-washing method may be a better choice. ${ }^{10}$ When correcting hand-washing defects, we found that insufficient frictional force and small contact surface area between the palm and back of the hand were prone to defects during washing of back of the hands and the back of the fingers. The effectiveness of training subjects resulted in significantly improved hand washing technique of the back of the hands, finger, and the wrists. This improvement decreased the hand-washing defect rate by $18 \%, 7.9 \%$, and $11.1 \%$, respectively. Regarding the handwashing defect in other parts of the hand, no significant difference was found before the training and on the first day after the training, which is presumably associated with the trainer's teaching abilities. Regarding the handwashing defects in the back of the fingers and the wrists, the day-2 post-training washing had a significantly higher defect rate than the day-1 post-training washing (19.6\% vs $7.9 \%$ and $26.5 \%$ vs $11.1 \%$, respectively). These results suggest that the training compliance gradually weakens over time; therefore training should be repeated regularly to ensure long-term compliance and good practice reinforcement. For the finger-tip back is more prone to hand washing defects, we consider that it is also related to the hand movement habits, these areas are not commonly used in daily contact, but hand washing defects can easily lead to infection when touching the nose, mouth and eyes. Therefore, it is necessary to pay attention to the hand washing process of these parts during hand washing. But the skin on the back of the hand is extremely sensitive to irritation, and if hand washing is strictly enforced multiple times especially with soapy water, the epidermal barrier may be disrupted, so we need to wash gently. Although PAC can be a good reminder of hand washing defects, whether this method can really improve the performance of hand hygiene in clinical practice and life remains to be further studied.

In the current study, we selected PAC as the tracer for several reasons: (1) its strong adsorption because of its rich pore-like structures. (2) it's extremely high biosecurity for clinical practice. ${ }^{32}$ In addition, PAC is often available in the pharmacy and Emergency room of health care institutions; and it can easily be obtained from the online store. Therefore, it can safely and conveniently be used as a tracer in hand-washing training to correct hand-washing defects. This is especially relevant considering the current environment and the COVID-19 pandemic.

In order to ensure hand washing compliance, it was necessary to survey the acceptance of the trainees toward hand hygiene training. A questionnaire survey was considered a good feedback tool for examining training acceptance and effectiveness. ${ }^{33}$ According to the survey results, hand washing training that utilized PAC performed well in terms of deepening subjects' interests and understanding of hand-washing, therefore helping improve hand-washing technique. This positive feedback result could be credited to the real-time visual display of the PAC tracing method to hand-washing defects. Visualized teaching and training through the implementation of tracers help trainers and the trainees to see the errors that occur during the training, and by this provide appropriate solutions to the errors. In this study, PAC performed noticeably well in achieving the goal of highlighting the errors that occur during the hand-washing process. Subjects also reported very few side effects associated with PAC, with satisfactory high acceptance rate. The main complaint conveyed by the subjects was the deposition of carbon residues in the nail grooves. We intend to use this method in the future for hand hygiene training. In order to confirm the efficacy and the results we found in the initial training while using the PAC tracer, we have carried out bacterial cultures of different parts of the hand 
after the hand hygiene training. The results from the bacterial culture will be used to improve the hand washing training process as by PDCA principle.

Despite all its merits, this study is not without its limitations. The particle size of PAC in different medical institutions is not the same, so it remains unclear whether a uniform training outcome can be achieved. In addition, this study cannot verify whether the areas with PAC residues can also be correlated as areas with probable bacteria; therefore, there is a need for further investigation by methods like the use of bacterial culture.

\section{Conclusions}

PAC tracing method can visually display hand-washing defects, thereby significantly improving the quality of hand-washing training. The back of the hand is more prone to hand-washing defects. The training method had a good acceptance rate, as well as a good safety profile.

\section{Funding}

This work was supported by the Science Foundation of Liaoning Education Department (FWZR2020012); 345 Talent Project of Shengjing Hospital.

\section{Disclosure}

The authors report no conflicts of interest related to this work.

\section{References}

1. Watkins J. Preventing a covid-19 pandemic. BMJ. 2020;368:m810. doi:10.1136/bmj.m810

2. Chu J, Yang N, Wei Y, et al. Clinical characteristics of 54 medical staff with COVID-19: a retrospective study in a single center in Wuhan, China. J Med Virol. 2020;92(7):807-813. doi:10.1002/jmv.25793

3. Lake MA. What we know so far: COVID-19 current clinical knowledge and research. Clin Med (Lond). 2020;20(2):124-127. doi:10.7861/clinmed.2019-coron

4. Mehta Y, Gupta A, Todi S, et al. Guidelines for prevention of hospital acquired infections. Indian J Crit Care Med. 2014;18(3):149-163. doi:10.4103/0972-5229.128705

5. Allegranzi B, Pittet D. Role of hand hygiene in healthcare-associated infection prevention. $J$ Hosp Infect. 2009;73(4):305-315. doi:10.1016/ j.jhin.2009.04.019

6. Adhikari SP, Meng S, Wu Y-J, et al. Epidemiology, causes, clinical manifestation and diagnosis, prevention and control of coronavirus disease (COVID-19) during the early outbreak period: a scoping review. Infect Dis Poverty. 2020;9(1):29. doi:10.1186/s40249-02000646-x

7. Kampf G, Brüggemann Y, Kaba HEJ, et al. Potential sources, modes of transmission and effectiveness of prevention measures against SARS-CoV-2. J Hosp Infect. 2020;106(4):678-697. doi:10.1016/j. jhin.2020.09.022
8. World Health OrganizationCopyright (C) 2009. WHO guidelines approved by the guidelines review committee, in WHO guidelines on hand hygiene in health care: first global patient safety challenge clean care is safer care. Geneva: World Health Organization; 2009.

9. Kratzel A, Todt D, V'kovski P, et al. Inactivation of severe acute respiratory syndrome coronavirus 2 by WHO-recommended hand rub formulations and alcohols. Emerg Infect Dis. 2020;26(7):1592-1595. doi:10.3201/eid2607.200915

10. Kwok YL, Callard M, McLaws ML. An automated hand hygiene training system improves hand hygiene technique but not compliance. Am J Infect Control. 2015;43(8):821-825. doi:10.1016/ j.ajic.2015.04.201

11. Kutafina E, Laukamp D, Bettermann R, et al. Wearable sensors for eLearning of manual tasks: using forearm EMG in hand hygiene training. Sensors (Basel). 2016;16(8):1221. doi:10.3390/s16081221

12. Higgins A, Hannan MM. Improved hand hygiene technique and compliance in healthcare workers using gaming technology. $J$ Hosp Infect. 2013;84(1):32-37. doi:10.1016/j.jhin.2013.02.004

13. Kumar JK, Patthi B, Singla A, et al. Graphical assessment technique (GAT) - an objective, comprehensive and comparative hand hygiene quantification tool. $J$ Clin Diagn Res. 2016;10(8):Zc118-22. doi:10.7860/JCDR/2016/18791.8371

14. Eggers M, Koburger-Janssen T, Ward LS, et al. Bactericidal and virucidal activity of povidone-iodine and chlorhexidine gluconate cleansers in an in vivo hand hygiene clinical simulation study. Infect Dis Ther. 2018;7(2):235-247. doi:10.1007/s40121-018-0202-5

15. Kac G, Podglajen I, Gueneret M, et al. Microbiological evaluation of two hand hygiene procedures achieved by healthcare workers during routine patient care: a randomized study. J Hosp Infect. 2005;60 (1):32-39. doi:10.1016/j.jhin.2004.10.014

16. Björklund K, Li LY. Adsorption of organic stormwater pollutants onto activated carbon from sewage sludge. J Environ Manage. 2017;197:490-497. doi:10.1016/j.jenvman.2017.04.011

17. van Doremalen N, Bushmaker T, Morris DH, et al. Aerosol and surface stability of SARS-CoV-2 as compared with SARS-CoV-1. $N$ Engl $J \quad$ Med. 2020;382(16):1564-1567. doi:10.1056/ NEJMc2004973

18. Chin AWH, Chu JTS, Perera MRA, et al. Stability of SARS-CoV-2 in different environmental conditions. Lancet Microbe. 2020;1(1):e10. doi:10.1016/S2666-5247(20)30003-3

19. Chan JF, Yuan S, Kok KH, et al. A familial cluster of pneumonia associated with the 2019 novel coronavirus indicating person-toperson transmission: a study of a family cluster. Lancet. 2020;395 (10223):514-523. doi:10.1016/S0140-6736(20)30154-9

20. Glauser W. Proposed protocol to keep COVID-19 out of hospitals. CMAJ. 2020;192(10):E264-e265. doi:10.1503/cmaj.1095852

21. Boyce JM. Current issues in hand hygiene. Am J Infect Control. 2019;47s:A46-a52. doi:10.1016/j.ajic.2019.03.024

22. Trampuz A, Widmer AF. Hand hygiene: a frequently missed lifesaving opportunity during patient care. Mayo Clin Proc. 2004;79 (1):109-116. doi:10.4065/79.1.109

23. Chen P, Yuan T, Sun Q, et al. Role of quality control circle in sustained improvement of hand hygiene compliance: an observational study in a stomatology hospital in Shandong, China. Antimicrob Resist Infect Control. 2016;5:54. doi:10.1186/s13756-016-0160-1

24. Shen L, Wang X, An J, et al. Implementation of WHO multimodal strategy for improvement of hand hygiene: a quasi-experimental study in a Traditional Chinese Medicine hospital in Xi'an, China. Antimicrob Resist Infect Control. 2017;6:98. doi:10.1186/s13756017-0254-4

25. Huang L, Lin G, Tang L, et al. Special attention to nurses' protection during the COVID-19 epidemic. Crit Care. 2020;24(1):120. doi:10.1186/s13054-020-2841-7

26. Salati SA, Al Kadi A. Hand hygiene practices in medical students: a follow-up study. Int Sch Res Notices. 2014;2014:591879. 
27. Li Y, Wang Y, Yan D, et al. Self-reported hand hygiene practices, and feasibility and acceptability of alcohol-based hand rubs among village healthcare workers in Inner Mongolia, China. J Hosp Infect. 2015;90(4):338-343. doi:10.1016/j.jhin.2015.04.006

28. Sadeghi-Moghaddam P, Arjmandnia M, Shokrollahi M, et al. Does training improve compliance with hand hygiene and decrease infections in the neonatal intensive care unit? A prospective study. $J$ Neonatal Perinatal Med. 2015;8(3):221-225. doi:10.3233/NPM15915001

29. Gould DJ, Moralejo D, Drey N, et al. Interventions to improve hand hygiene compliance in patient care. Cochrane Database Syst Rev. 2017;9(9):Cd005186.

30. Lacey G, Zhou J, Li X, et al. The impact of automatic video auditing with real-time feedback on the quality and quantity of handwash events in a hospital setting. Am J Infect Control. 2020;48 (2):162-166. doi:10.1016/j.ajic.2019.06.015
31. Goodsell DS, Jenkinson J. Molecular illustration in research and education: past, present, and future. J Mol Biol. 2018;430 (21):3969-3981. doi:10.1016/j.jmb.2018.04.043

32. Chakravarthi A, Srinivas CR, Mathew AC. Activated charcoal and baking soda to reduce odor associated with extensive blistering disorders. Indian J Dermatol Venereol Leprol. 2008;74(2):122-124. doi:10.4103/0378-6323.39694

33. Modi PD, Kumar P, Solanki R, et al. Hand hygiene practices among Indian medical undergraduates: a questionnaire-based survey. Cureus. 2017;9(7):e1463.
Risk Management and Healthcare Policy

\section{Publish your work in this journal}

Risk Management and Healthcare Policy is an international, peerreviewed, open access journal focusing on all aspects of public health, policy, and preventative measures to promote good health and improve morbidity and mortality in the population. The journal welcomes submitted papers covering original research, basic science, clinical \& epidemiological studies, reviews and evaluations,

\section{Dovepress}

guidelines, expert opinion and commentary, case reports and extended reports. The manuscript management system is completely online and includes a very quick and fair peer-review system, which is all easy to use. Visit http://www.dovepress.com/testimonials.php to read real quotes from published authors. 